\title{
THE CLASS GROUP OF DEDEKIND DOMAINS
}

\author{
BY \\ C. R. LEEDHAM-GREEN
}

\begin{abstract}
A new proof is given of Claborn's theorem, namely that every abelian group is the class group of a Dedekind domain. A variation of the proof shows that the Dedekind domain can be constructed to be a quadratic extension of a principal ideal ring; a Dedekind domain is also constructed that is unrelated in a certain sense to any principal ideal ring.
\end{abstract}

Introduction. Claborn proved in [2] that every abelian group is the class group of some Dedekind domain. In $\S 1$ a new proof is given which is based on a naive geometrical construction. In $\$ 2$ the construction is embellished to show that the Dedekind domain can appear as a quadratic extension of a principal ideal ring; a Dedekind domain is also constructed which is unrelated to any principal ideal ring; for precise statements see Theorems 2.1 and 2.3. The last result answers a problem due to Claborn [1].

1. Every abelian group is a class group. A well-known characterisation of a Dedekind domain as an intersection of valuation rings will be used. A valuation on a field $F$ will mean a normalized exponential valuation; that is to say a homomorphism $v$ of the multiplicative group of $F$ onto the integers under + satisfying $v(a+b) \geqq \min \{v a, v b\}$, with the convention $v 0=\infty$. In the notation of Weiss [3] an ordinary arithmetic field is a field $F$ and a nonempty family $\left\{v_{P} \mid P \in \Lambda\right\}$ of valuations of $F$ satisfying

(1.1) $\forall a \in F, v_{P} a \geqq 0$ for almost all $P \in \Lambda$;

(1.2) $\forall P_{1} \neq P_{2} \in \Lambda, \exists a \in F$ s.t. $v_{P_{1}}(a-1) \geqq 1 ; v_{P_{2}} a \geqq 1 ; \forall P \in \Lambda, v_{P} a \geqq 0$.

Then $D=\left\{a \in F \mid \forall P \in \Lambda, v_{P} a \geqq 0\right\}$ is a Dedekind domain. Denoting again by $P$ the set $\left\{a \mid a \in D, v_{P} a>0\right\}, P$ is a prime ideal of $D$ and every nonzero prime ideal of $D$ is of this form; so $\Lambda$ freely generates the group of (fractional) ideals of $D$. The field of fractions of $D$ is clearly $F$.

The class groups of the Dedekind domains we shall construct appear as follows. The generators, or prime ideals, will correspond to certain points in an affine plane, and the relators will correspond to the algebraic curves in that plane; a generator will occur with multiplicity $n$ in the relator corresponding to a given curve if the curve passes through the corresponding point with multiplicity $n$. We shall therefore need

Received by the editors December 8, 1970.

AMS 1970 subject classifications. Primary 13D15, 13 F05.

Key words and phrases. Class group, Dedekind domain.

Copyright (C) 1972, American Mathematical Society 
Lemma 1.1. Let $K$ be a perfect infinite field, $\bar{K}$ be an algebraic closure of $K$, and $A$ be an affine plane over $\bar{K}$. Let $\Lambda$ be a set of cardinal less than that of $K,\left\{P_{\lambda} \mid \lambda \in \Lambda\right\}$ be a family of points in A pairwise nonconjugate over $K$, and $\left\{n_{\lambda} \mid \lambda \in \Lambda\right\}$ be a family of nonnegative integers, nearly all zero. Then there is an algebraic curve $C$ in $A$, defined and irreducible over $K$, which, for all $\lambda$ in $\Lambda$, passes through $P_{\lambda}$ with multiplicity exactly $n_{\lambda}$. (Two points are conjugate over $K$ if some automorphism of $\bar{K}$ over $K$ takes one point to the other.)

Proof. The algebraic curves in $A$ which are defined over $K$ and, for all $\lambda$ in $\Lambda$, pass through $P_{\lambda}$ with multiplicity at least $n_{\lambda}$ form a projective space over $K$; and for any $m \geqq 0$ those of degree at most $m$ form a finite-dimensional subspace $T_{m}$. For each $\lambda$, the algebraic curves which pass through $P_{\lambda}$ with multiplicity at least $n_{\lambda}+1$ and for all $\mu$ pass through $P_{\mu}$ with multiplicity at least $n_{\mu}$ form a subspace $T_{m \lambda}$ of $T_{m}$. The curves in $T_{m}$ which are reducible over $K$ form a subvariety $V_{m}$ of $T_{m}$. It is not hard to verify that, for $m$ large enough, $T_{m \lambda}$ and $V_{m}$ are proper subvarieties of $T_{m}$. Since $\Lambda$ is of cardinal less than the (infinite) cardinal of $K$, it follows that, for $m$ large enough, there is a curve $C$ in $T_{m}$ which does not lie in $\left(\bigcup_{\lambda \in \Lambda} T_{m \lambda}\right) \cup V_{m}$.

The next result reduces the construction to the selection of a suitable family of points in an affine plane.

Proposition 1.2. Let $K$ be a perfect field and $A$ be an affine plane with coordinates $s$ and $t$ over an algebraic closure $\bar{K}$ of $K$. Let $\left\{P_{\lambda} \mid \lambda \in \Lambda\right\}$ be a family of points of $A$, pairwise nonconjugate over $K$, such that no algebraic curve (over $K$ ) passes through an infinite number of these points. Let $D$ be the subset of $K(s, t)$ consisting of all elements $f / g$ where $f, g$ in $K[s, t]$ are such that the multiplicity of $f$ at $P$ is not less than the (possibly zero) multiplicity of $g$ at $P$. Then $D$ is a Dedekind domain containing $K[s, t]$.

Moreover, if $\mathfrak{A}(\Lambda)$ is the free abelian group on $\Lambda$, and if $\left\{C_{i} \mid i \in I\right\}$ is the set of algebraic curves in $A$ defined and irreducible over $K$, then the class group of $D$ is isomorphic to $\mathfrak{A}(\Lambda)$ divided out by the relators $\left\{\sum_{\lambda \in \Lambda} n_{i \lambda} \lambda \mid i \in I\right\}$ where $n_{i \lambda}$ is the multiplicity of $C_{i}$ at $P_{\lambda}$.

Proof. For fixed $\lambda$ in $\Lambda$, and any $f$ in $K[s, t]$, let $v_{\lambda} f$ be the multiplicity of $f$ at $P_{\lambda}$. Then $v_{\lambda}$ extends uniquely to a valuation of $K(s, t)$ which we also denote by $v_{\lambda}$. Then for the first statement it is enough to prove that $K(s, t)$, together with $\left\{v_{\lambda} \mid \lambda \in \Lambda\right\}$, is an ordinary arithmetic field. Condition (1.1) is satisfied since every algebraic curve passes through only a finite number of the points $P_{\lambda}$. Now let $\lambda, \mu$ be distinct elements of $\Lambda$. Since $P_{\lambda}$ and $P_{\mu}$ are not conjugate over $K$, there is an element $f$ of $K[s, t]$ which defines a curve passing through $P_{\lambda}$ but not through $P_{\mu}$. Clearly we may choose $f$ such that $f\left(P_{\mu}\right)=1$. Then $v_{\lambda} f \geqq 1, v_{\mu}(f-1) \geqq 1$, and for all $\nu$ in $\Lambda, v_{v} f \geqq 0$. Thus condition (1.2) is satisfied and $D$ is a Dedekind domain whose $\mathrm{i}^{\prime}$. zal group is naturally isomorphic to $\mathfrak{X}(\Lambda)$. 
The group of principal ideals of $D$ is generated by those ideals which are generated by an irreducible element $f$ of $K[s, t]$. $f$ defines a curve $C_{i}, i \in I$; and the corresponding ideal is mapped by the above isomorphism to $\sum_{\lambda \in \Lambda}\left(v_{\lambda} f\right) \lambda$ in $\mathfrak{A}(\Lambda)$. But $v_{\lambda} f$ is the multiplicity $n_{i \lambda}$ of $C_{i}$ at $P_{\lambda}$. This proves the last statement of the proposition.

The coefficients of the above relators are all positive integers. We will thus need

Lemma 1.3. Let $G$ be an abelian group and $\Lambda$ be an infinite cardinal not less than the cardinal of $G$. Then $G$ can be presented as the quotient of a free abelian group on $\Lambda$ generators divided out by $\Lambda$ distinct nontrivial relators, each of which is a sum of nonnegative multiples of the free generators.

Proof. $G$ can certainly be presented as a quotient of a free abelian group of rank at most $\Lambda$ divided out by at most $\Lambda$ relators. Each relator will be of the form $p-q$, where $p$ and $q$ are sums of nonnegative multiples of the free generators. For each relator $p-q$, introduce a new free generator $x$, and replace the relator $p-q$ by $p+x$ and $q+x$. In this way $G$ can be expressed as the quotient of a free abelian group of rank at most $\Lambda$ divided out by at most $\Lambda$ relators, all of the required form. To ensure that there are exactly $\Lambda$ free generators and $\Lambda$ relators, add $\Lambda$ new generators and include them among the relators.

We now give our proof of Claborn's theorem. The proof can be simplified slightly by assuming that $K$ is algebraically closed, in which case problems of conjugacy no longer arise; however the more general case will be of interest when we indicate how the Dedekind domain can arise as a quadratic extension of a principal ideal ring.

THEOREM 1.4. Let $K$ be an infinite perfect field and $G$ be an abelian group of cardinal not greater than that of $K$. Let $s, t$ be algebraically independent over $K$. Then there is a Dedekind domain $D$ containing $K[s, t]$ with field of fractions $K(s, t)$ and class group isomorphic to $G$.

Proof. The idea of the proof is as follows. Using Proposition 1.2 it is enough to find a suitable set of points in an affine plane over $\bar{K}$. Given a presentation of $G$ as in Lemma 1.3, points are chosen to correspond to the given generators, and Lemma 1.1 is used to find curves which will produce relators. Unfortunately all the other irreducible algebraic curves passing through the given points will also produce relators, so further points are introduced which give generators whose sole purpose is to kill these unwanted relators. Points and curves corresponding to the given generators and relators will be called industrious, and those whose sole purpose is to kill each other off will be called otiose. We now begin the formal proof. Let $\Lambda$ be the least ordinal with cardinal equal to that of $K$. By Lemma 1.3, $G$ may be represented as the quotient of the free abelian group $\mathfrak{A}(\Lambda)$ on $\Lambda$ by distinct nontrivial relators $r_{\mu}, \mu \in \Lambda$, where $r_{\mu}=\sum_{\lambda \in \Lambda} n_{\mu \lambda} \lambda, n_{\mu \lambda} \geqq 0$ for all $\mu, \lambda$, and $n_{\mu \lambda}=0$ almost always. In this way both the generators and relators have been well ordered, but we only use the ordering of the former. If, for some $\mu, \lambda$ is the largest element of 
$\Lambda$ such that $n_{\mu \lambda}>0$ call $\lambda$ the leading term of $r_{\mu}$. Now let $\bar{K}$ be an algebraic closure of $K$, and $A$ be the affine plane over $\bar{K}$ with coordinates $s$ and $t$. Then the algebraic curves in $A$ which are defined and irreducible over $K$ may be indexed as $\left\{C_{\lambda} \mid \lambda \in \Lambda\right\}$. For each $\mu$ in $\Lambda$ we shall construct, by transfinite induction, a subset $\mathscr{C}_{\mu}$ of $\left\{C_{\lambda}\right\}$ and a pair of points $P_{\mu}, Q_{\mu}$ in $A$ such that

(i) $P_{\mu}$ and $Q_{\mu}$ are not conjugate to each other over $K$;

(ii) neither $P_{\mu}$ nor $Q_{\mu}$ is conjugate over $K$ to $P_{\nu}$ or $Q_{v}$ for any $\nu<\mu$;

(iii) neither $P_{\mu}$ nor $Q_{\mu}$ lies on any curve contained in $\bigcup_{\nu<\mu} \mathscr{C}_{v}$;

(iv) no curve of degree $n$ which passes through $n^{2}+1$ of the points $P_{v}, Q_{v}$, $\nu<\mu$, passes through $P_{\mu}$ or $Q_{\mu}$;

(v) $\mathscr{C}_{\mu}$ is obtained by adjoining to $\bigcup_{v<\mu} \mathscr{C}_{v}$

(a) the first irreducible curve, $C_{\alpha(\mu)}$ say, which is not already contained in $\bigcup_{v<\mu} \mathscr{C}_{v}$, and

(b) for every relator $r_{\tau}$ with leading term $\mu$ an irreducible curve $C_{\beta(\tau)}$ which passes through $P_{\nu}$ with multiplicity exactly $n_{\tau \nu}$ for all $\nu \leqq \mu$, and passes through no point $Q_{\nu}$ for $\nu \leqq \mu$;

(vi) $Q_{\mu}$ is a simple point of $C_{\alpha(\mu)}$.

The points $P_{\mu}$ and curves $C_{\beta(\tau)}$ will be industrious while $Q_{\mu}$ and $C_{\alpha(\mu)}$ will be otiose. Condition (iii) ensures that once a relator has been dealt with it does not subsequently change. Condition (iv) ensures that no curve (in particular no $C_{\alpha(u)}$ ) passes through infinitely many of the points $P_{\lambda}, Q_{\lambda}$. If $\Lambda$ is countable this has already been ensured by (iii). Condition (v)(a) ensures that every irreducible curve appears in $\mathscr{C}_{\mu}$ for $\mu$ large enough; observe that the curves adjoined in (v) do not already lie in $\bigcup_{v<\mu} \mathscr{C}_{v}$ since they pass through $P_{\mu}$ or $Q_{\mu}$, and that $C_{\alpha(\mu)}$ is not among the $C_{\beta(\tau)}$ since it passes through $Q_{\mu}$.

To check that the construction is possible, suppose that for all $\nu<\mu, P_{v}, Q_{v}$, and $\mathscr{C}_{v}$ have been chosen to satisfy the above conditions. $C_{\alpha(\mu)}$ is determined by (v)(a). Now choose $P_{\mu}$ and $Q_{\mu}$. Condition (vi) reduces the choice of $Q_{\mu}$ to the $\Lambda$ simple points of $C_{\alpha(\mu)}$. Since the cardinal of $\mu$ is less than that of $\Lambda$, conditions (ii) and (iii) exclude less than $\Lambda$ of these points. Since, by Bezout's theorem, there is at most one irreducible curve of degree $n$ through $n^{2}+1$ given points of $A$, the points excluded by condition (iv) lie on less than $\Lambda$ irreducible curves, and so again less than $\Lambda$ of the simple points of $C_{\alpha(\mu)}$ are excluded. Thus there are $\Lambda$ possible choices for $Q_{\mu}$, and similarly for $P_{\mu}$, which satisfy (ii), (iii), (iv) and (vi), and so $P_{\mu}$ and $Q_{\mu}$ can be chosen to satisfy these conditions and not be conjugate over $K$ (condition (i)). Finally, the curves $C_{\beta(\tau)}$ may be chosen to satisfy (v)(b) by Lemma 1.1.

Now apply Proposition 1.2 with $\left\{P_{\lambda}\right\}$ replaced by $\left\{P_{\lambda}\right\} \cup\left\{Q_{\lambda}\right\}$. It follows from the above remarks that the hypotheses of the proposition are satisfied and so a Dedekind domain $D$ is obtained. The points $P_{\lambda}$ and $Q_{\lambda}$ may be regarded as generators of the class group of $D$, relators being supplied as in Proposition 2.2 by the irreducible curves in $A$. By (iii) and (v)(b), the relator defined by an industrious curve $C_{\beta(\tau)}$ only involves generators $P_{\lambda}$ whereas the relator defined by an otiose 
curve $C_{\alpha(\mu)}$ involves $Q_{\mu}$ with multiplicity one and no $Q_{\nu}$ for $\nu>\mu$. It follows that the otiose points and curves do indeed kill each other off, and we are left with a class group with the given presentation.

As a final remark, the group of units of $D$ is easily calculated. The presentation of $G$ chosen at the beginning of the proof gives an exact sequence $0 \rightarrow X \rightarrow Y$ $\rightarrow Z \rightarrow G \rightarrow 0$, where $Z$ is the free abelian group on the given generators and $Y$ is the free abelian group on the given relators. Then the group of units of $D$ is isomorphic to the direct product of the multiplicative group of $K$ with $X$. Of course the presentation may be chosen so that $X$ is trivial.

2. Relations with principal ideal rings. After some prefatory remarks about extensions of Dedekind domains we prove first that every abelian group is the class group of a quadratic extension of a principal ideal ring.

If $L$, with a set of valuations $\left\{v_{P} \mid P \in \Lambda\right\}$, is an ordinary arithmetic field, and $M$ is a finite algebraic extension of $L$, then $M$, with the set of all extensions to $M$ of the valuations $v_{P}$, is an ordinary arithmetic field; and if $O_{L}$ and $O_{M}$ are the corresponding Dedekind domains then $O_{M}$ is the integral closure of $O_{L}$ in $M$. The problem is to arrange matters so that $O_{L}$ is a principal ideal ring, $O_{M}$ has pre-assigned class group, and $M$ is a quadratic extension of $L$. Let $K$ be a quadratic extension of $F$; and to simplify matters slightly, suppose that $K$ is algebraically closed (so that $K$ is of characteristic zero and $F$ is real-closed). Take $L$ and $M$ above to be $F(s, t)$ and $K(s, t)$ respectively. $F(s, t)$ may be made into an ordinary arithmetic field as in $\S 1$ by selecting a suitable set of points in an affine plane $A$ over $K$, distinct points being nonconjugate over $F$. If $v$ is the valuation of $F(s, t)$ corresponding to a point $P$ in $A$, the extensions of $v$ to $K(s, t)$ correspond to $P$ and its conjugate $P^{\prime}$. Bear in mind that since every curve defined over $F$ which passes through one of $P$ and $P^{\prime}$ will also pass through the other, $P$ and $P^{\prime}$ define the same valuation of $F(s, t)$, but different valuations of $K(s, t)$ unless of course $P=P^{\prime}$. Observe also that since $K$ is a separable extension of $F$ no prime ramifies; this comes down to the fact that given any point $P$ in $A$ there is a curve defined over $F$ which passes through $P$ with multiplicity one.

TheORem 2.1. Let $G$ be an abelian group. Then there is a principal ideal ring $O$ with field of fractions $L$, and a quadratic extension $M$ of $L$, such that the integral closure of $O$ in $M$ has class group $G$.

Proof. $L$ and $M$ will be $F(s, t)$ and $K(s, t)$ with $F$ and $K$ as above, and $D$, the integral closure of $O$ in $M$, will be constructed along the lines of the proof of Theorem 1.4. When $D$ is constructed, $O=D \cap F(s, t)$ is also determined. There are two main adjustments to be made to the earlier proof. Firstly, whenever a point in $A$ is chosen so must its conjugate over $F$. Secondly, one must ensure that the class group of $O$ will be trivial. This will come about from the fact that conjugate points over $F$ define the same valuation of $F(s, t)$, so pairs of generators become equated. However, relators arising from curves that are not defined over $F$ will be lost. To 
avoid duplication only one of each pair of conjugate points will be used at first, the conjugates being added later. Instead of adding in an industrious and an otiose point at the $\mu$ th stage of the construction, whenever $C_{\alpha(\mu)}$ is not defined over $F$ only an otiose point will be introduced, and whenever $C_{\alpha(\mu)}$ is defined over $F$ only an industrious point will be introduced, its conjugate supplying the required otiose point. For this reason it will be convenient to use the same letter for each kind of point. We now begin the formal proof; conjugacy will always be over $F$. Let $F$ be a real-closed field with cardinal at least as great as the cardinal of $G$, and $\Lambda$ be the least ordinal of that cardinal. Let $A$ be the affine plane over an algebraic closure $K$ of $F$ with coordinates $s$ and $t$. Index the algebraic curves in $A$ which are defined and irreducible over $K$ as $\left\{C_{\lambda} \mid \lambda \in \Lambda\right\}$. A strictly increasing function $\alpha: \Lambda \rightarrow \Lambda$ will be defined below with the property that every curve in $A$ that is defined over $F$ and irreducible over $K$ is of the form $C_{\alpha(\gamma)}$ for some $\gamma \in \Lambda$. Let $\Gamma$ be the subset of $\Lambda$ consisting of elements $\gamma$ such that $C_{\alpha(\gamma)}$ is defined over $F$. Then $\Gamma$ is of the same cardinal as $\Lambda$, and $G$ may be represented, as in Lemma 1.3, as the quotient of a free abelian group on $\Gamma$ by distinct nontrivial relators $r_{\mu}, \mu \in \Lambda$, where $r_{\mu}=\sum_{\gamma \in \Gamma} n_{\mu \gamma} \gamma$, and $n_{\mu, \gamma} \geqq 0$ for all $\mu, \gamma$. For each $\mu$ in $\Lambda$ we shall construct, by transfinite induction, a subset $\mathscr{C}_{\mu}$ of $\left\{C_{\lambda}\right\}$ and a point $P_{\mu}$ in $A$ such that

(i) the coefficients of $P_{\mu}$ do not both lie in $F$;

(ii) for all $\nu<\mu, P_{\mu}$ is neither equal nor conjugate to $P_{v}$;

(iii) neither $P_{\mu}$ nor its conjugate lies on any curve in $\bigcup_{v<\mu} \mathscr{C}_{v}$;

(iv) no curve of degree $n$ which passes through $n^{2}+1$ of the points $P_{v}$ and their conjugates, $\nu<\mu$, passes through $P_{\mu}$ or its conjugate;

(v) if the first irreducible curve, $C_{\alpha(\mu)}$ say, which is neither equal nor conjugate to a curve already contained in $\bigcup_{\nu<\mu} \mathscr{C}_{v}$, is not defined over $F$, then $\mathscr{C}_{\mu}$ is obtained by adjoining $C_{\alpha(\mu)}$ to $\bigcup_{\nu<\mu} \mathscr{C}_{\nu}$. Also, $P_{\mu}$ is a simple point of $C_{\alpha(\mu)}$ whose conjugate does not lie in $C_{\alpha(\mu)}$.

(vi) If $C_{\alpha(\mu)}$ is defined over $F$, so that $\mu \in \Gamma$, then $\mathscr{C}_{\mu}$ is obtained by adjoining to $\bigcup_{v<\mu} \mathscr{C}_{v}$ both $C_{\alpha(\mu)}$ and, for every relator $r_{\tau}$ with leading term $\mu$, an irreducible curve $C_{\beta(\tau)}$ which passes through $P_{\gamma}$ with multiplicity exactly $n_{\tau \gamma}$ for all $\gamma \in \Gamma$, $\gamma \leqq \mu$, and which passes through no other point $P_{v}, \nu<\mu$, and through the conjugate of no point $P_{v}, \nu \leqq \mu$. Also, $P_{\mu}$ is a simple point of $C_{\alpha(\mu)}$.

Every curve or its conjugate will appear in $\mathscr{C}_{\mu}$ for $\mu$ large enough. Moreover no curve adjoined in steps (v) and (vi) is conjugate to any curve already in $\bigcup_{v<\mu} \mathscr{C}_{v}$; and no two of the curves adjoined in (vi) are conjugate to each other, since $C_{\alpha(\mu)}$ is defined over $F$, and the curves $C_{B(\tau)}$ are not defined over $F$, their conjugates passing through no $P_{v}, \nu \leqq \mu$. Thus $\bigcup_{\lambda \in \Lambda} \mathscr{C}_{\lambda}$ contains exactly one element of every conjugacy class of irreducible curves over $K$. The function $\alpha$ is clearly increasing and has the other property claimed above since the curves $C_{\beta(\tau)}$ are not defined over $F$.

The above construction can be carried out as in the proof of Theorem 1.4. Write the conjugate of a point $P$ or a curve $C_{\lambda}$ as $P^{\prime}$ or $C_{\lambda}^{\prime}$. Now apply Proposition 1.2 with $\left\{P_{\lambda}\right\}$ replaced by $\left\{P_{\lambda}\right\} \cup\left\{P_{\lambda}^{\prime}\right\}$ and the $K$ of the proposition corresponding 
to the $K$ here. The hypotheses of the proposition are clearly satisfied and a Dedekind domain $D$ is obtained. The class group of $D$ is generated by elements $\lambda, \lambda^{\prime}$ corresponding to $P_{\lambda}$ and $P_{\lambda}^{\prime}, \lambda \in \Lambda$, with relators corresponding to the curves $C_{\alpha(\mu)}, C_{\beta(\tau)}$ and their conjugates, $\mu, \tau \in \Lambda$. If a curve $C$ passes through a point $P_{\lambda}$, then its conjugate passes through $P_{\lambda}^{\prime}$ with the same multiplicity; this holds in particular if $C$ is its own conjugate, i.e. if $C$ is defined over $F$. Using this and the fact that $\Lambda$ is well ordered, the relators corresponding to $C_{\alpha(\mu)}$ and $C_{\alpha(\mu)}^{\prime}, \mu \notin \Gamma$, can be used to replace the relators corresponding to $C_{\alpha(\gamma)}, \gamma \in \Gamma$, by new relators $s_{\gamma}$ satisfying

(a) only generators $\delta, \delta^{\prime}$, where $\delta \in \Gamma, \delta \leqq \gamma$, are involved;

(b) for all $\delta \leqq \gamma, \delta$ and $\delta^{\prime}$ appear with the same multiplicity;

(c) $\gamma$ and $\gamma^{\prime}$ appear with multiplicity 1 .

All generators $\lambda, \lambda^{\prime}$ and relators corresponding to $C_{\alpha(\lambda)}, C_{\alpha(\lambda)}^{\prime}, \lambda \notin \Gamma$, may now be ignored. There remain the generators $\gamma, \gamma^{\prime}, \gamma \in \Gamma$, and relators $s_{\gamma}, \gamma \in \Gamma, r_{\mu}=\sum_{\gamma} n_{\mu \gamma} \gamma$, and $r_{\mu}^{\prime}=\sum_{\gamma} n_{\mu \gamma} \gamma^{\prime}, \mu \in \Lambda$. The relators $s_{\gamma}$ may be used to eliminate the generators $\gamma^{\prime}$. Bearing in mind the original presentation of $G$, it must be verified that the relators $s_{\gamma}$ and $r_{\mu}^{\prime}$ do not imply any new relations $h^{-1}$ ween the elements of $\Gamma$. Suppose then that there are integers $a_{\gamma}, \gamma \in \Gamma, b_{\mu}, \mu \in \Lambda$, nearly all zero, such that $\sum a_{\gamma} s_{\gamma}+\sum b_{\mu} r_{\mu}^{\prime}$ involves only elements of $\Gamma$. Then by (b) above, $\sum a_{\gamma} s_{\gamma}+\sum b_{\mu} r_{\mu}^{\prime}+\sum b_{\mu} r_{\mu}=0$. Thus the relation implied on the elements of $\Gamma$ is a consequence of the relations $r_{\mu}=0$. Hence the class group of $D$ is isomorphic to $G$.

Now apply Proposition 1.2 again with $\left\{P_{\lambda}\right\}$ of the proposition corresponding to $\left\{P_{\lambda}\right\}$ here, but with $K$ replaced by $F$. The hypotheses of the proposition are again satisfied and a Dedekind domain $O$ is produced. The curves in $A$ which are defined and irreducible over $F$ are either irreducible over $K$, namely the curves $C_{\alpha(\lambda)}, \lambda \in \Gamma$, or split over $K$ into the union of two conjugate subvarieties, either $C_{\alpha(\lambda)} \cup C_{\alpha(\lambda)}^{\prime}$, $\lambda \notin \Gamma$, or $C_{\beta(\lambda)} \cup C_{\beta(\lambda)}^{\prime}, \lambda \in \Lambda$. Since the curves $C_{\alpha(\lambda)}, \lambda \in \Gamma$, and $C_{\alpha(\lambda)} \cup C_{\alpha(\lambda)}^{\prime}, \lambda \notin \Gamma$, pass through $P_{\lambda}$ with multiplicity 1 and through no point $P_{\mu}, \mu>\lambda$, the corresponding relators ensure that $O$ is a principal ideal ring. Moreover, by the remarks at the beginning of the paragraph, $D$ is the integral closure of $O$ in $K(s, t)$, and so the theorem is proved.

Our second variation of Theorem 1.4 goes to the opposite extreme. In [4], Zariski and Samuel asked whether every Dedekind domain is the integral closure of a principal ideal ring in a finite algebraic extensi $\mathrm{n}$ of its field of fractions, or, as we shall say, is a finite algebraic extension of a principal ideal ring. This was answered in the negative by Claborn in [1]; the idea of the counterexample was to take a principal ideal ring $O$, in fact the ring of rational integers, form a finite algebraic extension $E$ of $O$, and then consider the ring of fractions $D=E_{S}$ of $E$ with respect to a multiplicatively closed subset $S$ of $E$. Claborn therefore asked in [1] whether every Dedekind domain $D$ can be obtained from a principal ideal ring by constructing a finite algebraic extension and then forming a ring of fractions. Before answering this question, again in the negative, a simple 
LeMma 2.2. Let $E$ be an algebraic extension of degree $n$ of a principal ideal ring $O$, and $D$ be the ring of fractions of $E$ with respect to a multiplicatively closed subset $S$ of $E$. Then the set of prime ideals of $D$ may be partitioned into subsets of at most $n$ elements each such that a product of the primes in any subset, with suitable positive multiplicities, is a principal ideal.

Proof. Since the class group of $D$ is obtained from the class group of $E$ by dividing out by the prime ideals which intersect $S$, it is sufficient to take the case $D=E$. For every prime $p$ of $O$, let $T_{p}$ be the set of primes in $E$ dividing $p E$. Then the sets $T_{p}$ provide a partitioning as required.

It is now easy to prove

Theorem 2.3. Not every Dedekind domain $D$ may be constructed as follows. Take a principal ideal ring $O$, a finite algebraic extension $E$ of $O$, and put $D=E_{S}$, the ring of fractions of $E$ with respect to a multiplicatively closed set $S$.

A finite algebraic extension of a principal ideal ring was defined in the remarks before Lemma 2.2.

Proof. Adjust the construction of the Dedekind domain $D$ in the proof of Theorem 1.4 in the following ways. $K$ will be countable and algebraically closed, and $\Lambda$ will be the natural numbers. At the $m$ th stage $m$ points $P_{1}^{m}, \ldots, P_{m}^{m}$ will be constructed; there will be no analogue for $\mathscr{C}_{\mu}$. Conditions (i) to (vi) will be replaced by the requirements that whenever $1 \leqq i \leqq m$

(i) $P_{i}^{m}$ lies on no curve $C_{k}, k<m$;

(ii) $P_{i}^{m}$ is a simple point on $C_{m}$.

As in the proof of Theorem 1.4 a Dedekind domain $D$ is obtained. Now let $f$ and $g$ be coprime elements of $K[s, t]$ such that $f / g$ is in $D$, and let $s$ be the largest integer such that $C_{s}$ lies in the variety defined by $f$. Then since $f / g \in D, C_{t}$ does not lie in the variety defined by $g$ for any $t \geqq s$. Now suppose that $D$ arises from an algebraic extension $E$ of degree $n$ of a principal ideal ring $O$ as in the theorem. Take a partition of the primes of $D$ as in Lemma 2.2, and suppose that, in some subset $T_{p}$ in the partition, $s$ is the largest integer such that $P_{i}^{s}$ corresponds to a prime in $T_{p}$ for some $i$. Then it follows from the above remarks that the prime corresponding to $P_{j}^{s}$ is in $T_{p}$ for all $j, 1 \leqq j \leqq s$. But this implies $s \leqq n$, and hence a contradiction is achieved.

\section{BIBLIOGRAPHY}

1. L. Claborn, Dedekind domains and rings of quotients, Pacific J. Math. 15 (1965), 59-64. MR 31 \#2263.

2. —-, Every abelian group is a class group, Pacific J. Math. 18 (1966), 219-222. MR 33 \#4085.

3. E. Weiss, Algebraic number theory, McGraw-Hill, New York, 1963. MR 28 \#3021.

4. O. Zariski and P. Samuel, Commutative algebra. Vol. 1, University Series in Higher Math., Van Nostrand, Princeton, N. J., 1958. MR 19, 833.

Department of Pure Mathematics, Queen Mary College, london, England 